\title{
Factors Associated with Suicide: Case-Control Study in South Tyrol
}

\author{
by \\ Giancarlo Giupponi; ${ }^{\text {a Marco Innamorati; }}{ }^{b}$ Ross J. Baldessarini; ${ }^{c, d}$; Diego De Leo; ${ }^{\text {e }}$ \\ Francesca de Giovannelli a; Roger Pycha; ${ }^{f}$ Andreas Conca ${ }^{a}$; Paolo Girardi; Maurizio \\ Pompili ${ }^{c, g}$
}

From

a. Department of Psychiatry, Bolzano, Italy

b. European University of Rome, Italy;

c. International Consortium for Mood \& Psychotic Disorders Research, Mailman Research Center, McLean Hospital, Belmont, Massachusetts, USA;

d. Department of Psychiatry, Harvard Medical School, Boston, Massachusetts, USA;

e. Australian Institute of Suicide Research and Prevention and Griffith University, Mt Gravatt, Qld, Australia

f. Department of Psychiatry, Brunico, Italy

g. Department of Neurosciences, Mental Health and Sensory Organs, Suicide Prevention Center, Sant'Andrea Hospital, Sapienza University of Rome, Rome, Italy

Running title: Factors associated with suicide

Acknowledgments: Supported in part by a grant from the Bruce J Anderson Foundation and by the McLean Private Donors Psychiatric Research Fund (to RJB).

Disclosures: No author or immediate family member has financial relationships with commercial organizations that might appear to represent potential conflicts of interest with the material presented.

Submitted to: Comprehensive Psychiatry as a research report, June 2017, revised as COMPRPSYCHIATRY-D-17-00162, September 2017 [185 word abstract, 2078 word text, 47 references, 2 tables].

Correspondence to: Prof. M. Pompili: Department of Psychiatry, Sant'Andrea Hospital, 1039, Via di Grottarossa, 1035, Italy. Tel.: +39 06 33775675; Fax: +39 06 33775342; E-mail:

maurizio.pompili@uniroma1.it 


\begin{abstract}
Background: As suicide is related to many factors in addition to psychiatric illness, broad and comprehensive risk-assessment for risk of suicide is required. This study aimed to differentiate nondiagnostic risk factors among suicides versus comparable psychiatric patients without suicidal behavior. Methods: We carried out a pilot, case-control comparison of 131 cases of suicide in South Tyrol matched for age and sex with 131 psychiatric controls, using psychological autopsy methods to evaluate differences in clinically assessed demographic, social, and clinical factors, using bivariate conditional Odds Risk comparisons followed by conditional regression modeling controlled for ethnicity.

Results: Based on multivariable conditional regression modeling, suicides were significantly more likely to have experienced risk factors, ranking as: [a] family history of suicide or attempt $\geq[\mathrm{b}]$ recent interpersonal stressors $\geq[\mathrm{c}]$ childhood traumatic events $\geq$ [d] lack of recent clinician contacts $\geq[\mathrm{e}]$ previous suicide attempt $\geq[\mathrm{f}]$ non-Italian ethnicity, but did not differ in education, marital status, living situation, or employment, nor by psychiatric or substance-abuse diagnoses.

Conclusions: Both recent and early factors were associated with suicide, including lack of recent clinical care, non-Italian cultural subgroup-membership, familial suicidal behavior, and recent interpersonal distress.
\end{abstract}

Key words: case-control study, psychological autopsy, risk factors, South Tyrol, suicide

\title{
Introduction
}

Suicide is a leading, international public health challenge and cause of death. Over 800,000 people die by suicide each year, and 20-30 times more attempt suicide [1]. Suicide accounts for $1.4 \%$ of all deaths worldwide and is the 15th leading cause of death overall, but second among persons aged 15-29 years [2]. Efforts to improve prevention of suicide have included many studies of risk factors associated with suicidal behavior, usually based on searching for differences between samples of suicides and nonsuicides $[3,4]$. Such studies include use of psychological autopsy, a procedure that evolved from forensic investigations aimed at clarifying causes of death and differentiating suicides from accidental deaths $[5,6]$. This procedure has limitations, sometimes including reliance on expert opinions rather than verifiable facts about possible events related to deaths of uncertain cause, including the role of psychiatric illness.

Presence of psychiatric illness, particularly a major mood disorder, is a leading risk factor for suicide [7-9], although most persons with mental illnesses never attempt suicide [10]. Other reported risk factors for suicidal behavior include alcohol abuse $[11,12]$, substance dependence [13,14], attention disorders [15], particular affective 
temperaments [16,17] or other personality traits [18], childhood trauma [19-21], and a family history of suicidal behavior [22-24].

Many studies attempting to predict risk of suicide have concentrated on particular factors, whereas efforts to estimate the relative importance or relationships of different risk factors, or the predictive power of multiple factors are less well developed $[4,25,26]$. Prediction of suicide is particularly challenging owing to the low prevalence of this outcome [4]. Nevertheless, it seems clear that improved prediction of suicidal behavior should take a range of risk factors into account, including both temporally proximal and distal factors, and attempt to evaluate their relative importance and interactions [4,24].

In view of limited efforts to evaluate multiple potential risk factors in a single study, we designed this pilot study to compare a groups of subjects determined to have died by suicide with comparable subjects with psychiatric illnesses but no known suicidal behavior, and matched for sex and age in a case-control design. The study involved a culturally diverse population of the alpine region of South Tyrol. We aimed to identify demographic, cultural, and clinical factors, in addition to psychiatric diagnoses, that differentiated cases from controls, and specifically considered possible effects of the timing of traumatic or stress-related events.

\section{Methods}

Study design

We investigated differences between persons who died by suicide and nonsuicidal comparison subjects in the Province of Bolzano (Bozen) in South Tyrol, Italy. Information was collected during a psychological autopsy study approved by the Public Health Department of the Autonomous Province of Bolzano. Informed consent was obtained from living participants or close family members of suicides for analysis and anonymous and aggregate reporting of findings.

\section{Suicide cases}

We collected data about all individuals who died by suicide between 2000 and 2009 in South Tyrol, as verified by forensic postmortem examinations. Suicides were excluded if they 
involved persons who did not reside in the province, if informed consent was refused by informant family members, or age was $<18$ years. We reported elsewhere comprehensive analysis of the suicide cases [27]. For the present study, we compared 131suicide cases matched with 131 living, nonsuicidal, psychiatric controls.

\section{Psychiatric controls}

Controls had been hospitalized during the same years, were similar in morbidity or illness severity with the suicides, and were alive at the time of the study. These 131 nonsuicidal psychiatric controls were hospitalized in departments of psychiatry within the province of Bolzano for the first time between 2000 and 2009, selected at random and matched 1:1 for age (within \pm 2 years) and sex with the suicide cases, and still living at the close of the study in May, 2016. Cases and controls were not matched for psychiatric illness, but nonsuicidal, recently hospitalized psychiatric controls were similar to the suicide cases in the types and severity of psychiatric illnesses. We excluded candidate controls whose socio-demographic or clinical information was unavailable or when we could not ascertain whether the patient was currently alive.

\section{Data collection}

We asked clinicians from all Public Health Departments of South Tyrol to complete questionnaires regarding the premorbid conditions and causes and methods of death, as reported by family and death certificates, of all suicides occurring in 2000-2009. Of a total of 450 candidate suicide cases, 131 (29.1\%) were selected at random and as having adequate medical, psychiatric, and forensic records for review. Data so-obtained were cross-referenced with the provincial health database (MENTA, based on electronic clinical records), which provides socio-demographic information of province residents. We then conducted psychological autopsies based on structured enquiry into the following areas: [1] demographic factors, including education, employment, marital status, and living arrangements; [2] psychosocial and stress-related factors, such as major losses (including the occurrence and timing of separation or divorce, death of a spouse or partner, death or divorce of a parent or child), school or work failures, financial problems, and childhood 
traumas; [3] clinical information, including personal and family psychiatric history, including suicidal behaviors among first- or second-degree relatives, and patient helpseeking behavior (recent contact with a physician, psychologist or counselor); and [4] current psychiatric diagnosis. The autopsy procedure required information from least two informants, who included parents, other relatives or close friends, as well as information provided in medical records and from the public health questionnaires.

These psychological autopsy methods have been tested for reliability and validity, and have yielded consistently high concordance with direct clinical assessments $(\kappa=0.8-$ 0.9) $[28,29]$. We also found similar levels of inter-rater agreement regarding major psychiatric diagnoses [30,31].

If a psychiatric diagnosis was not available, informants were interviewed, and information about previous psychiatric assessments and treatment was collected from medical records and analyzed independently by two author-investigators (GG, RP) following SCID I and II interview schedules for DSM [32]. Required data regarding living controls were collected by two other experienced clinical investigators (FdG, MS) using medical records and other reports from clinicians. When diagnosis was not agreed-upon by both primary assessors, a third, experienced clinical investigator was consulted and all three worked to DSM-IV-TR-based, consensus diagnoses of any Axis I and Axis II disorder prior to suicide, and in the controls. In this investigation, the mean $\kappa$ value for inter-rater agreement on psychiatric diagnosis was 0.90 .

\section{Statistical analysis}

Analyses were performed with the Statistical Package for Social Sciences (SPSS.19.0 for Windows; IBM-SPSS Software, Armonk, NY). Descriptive data are reported as means \pm standard deviation (SD). Bivariate analyses included McNemar's and marginal homogeneity tests for paired nominal data. Conditional Odds Risk statistics (COR; [case exposed + control unexposed] / [case unexposed + control exposed]) are reported as measures of relative risk among suicide cases versus controls. Variables found to differ significantly between cases and controls (two-tailed $p<0.05$, uncorrected for multiple comparisons) in bivariate analyses were 
then included in a multi-variable conditional logistic regression. Resulting associations are reported as Odds Ratios (OR) with their $95 \%$ confidence intervals $(\mathrm{CI})$ and $p$-values. We used fully conditional specification (FCS) multiple imputation methods to impute the partially observed variables [33], using 5 imputations and a maximum of 10 iterations. Relative efficiency (i.e., the ratio of variance of a parameter given estimation with five imputations to variance if imputations were infinite) coefficients were close to 1 (all $\geq 0.97)$, indicating that the five chosen number of imputations was satisfactory. Binary variables were imputed using logistic regression.

\section{Results}

Subjects

After matching for age and sex, the study sample included 262 total subjects: 131 cases of suicide (81 men and 50 women; mean age $48.2 \pm 15.5$ years, and 131 controls $(81$ men and 50 women; mean age $48.3 \pm 15.4$ years (Table 1 ).

[Table 1 about here]

\section{Comparisons of suicide cases versus psychiatric controls}

The cases and controls were similar in most sociodemographic characteristics except for ethnicity (Table 1). Suicide cases were less often of the predominant regional Italian ethnicity (11.5\% of cases vs. $21.4 \%$ of controls), and more often of Austro-Germanic (75.6\% vs. $72.5 \%$ ), Ladin (4.6\% vs. $2.3 \%$ ), or other minority cultures (8.4\% vs. 3.8\%). Cases and controls were not formally matched for DSM-IV-TR psychiatric diagnoses, but did not differ in rates of these diagnoses (Table 1).

Single factors that were significantly more prevalent among suicide cases than controls ranked by relative risk (Table 1) as: [a] family history of suicidal behavior (2.86times); [b] recent interpersonal stressors (romantic disappointment or intrafamilial distress or discord within 6 months, usually related to major losses [2.44-fold]); [c] childhood traumatic experiences (2.40-times); [d] not having contact with a clinician within the past 4 weeks (2.33-fold); [e] previous suicide attempts (2.25-fold); and [f] nonItalian ethnicity (2.17-times). 


\section{Conditional regression modeling}

Multi-variable conditional regression modeling (Table 2) considered factors found to differ between cases and controls in bivariate comparisons (Table 1). Factors found to be significantly associated with suicide ranked by risk (Table 2) as: [a] non-Italian ethnicity $(\mathrm{OR}=3.36$ [CI: 1.35-8.37]); [b] recent major interpersonal loss or distress (OR = 2.70 [CI: 1.35-5.40]); [c] family suicidal history (OR = 2.67 [CI: 1.10-6.50]); [d] lack of recent clinical contact (OR $=2.57$ [CI: $1.22-5.44])$; and [e] childhood trauma (OR $=2.54$ [CI: $1.22-$ 5.23]).

[Table 2 about here]

\section{Discussion}

This study considered socio-demographic and clinical characteristics of an unusual, somewhat geographically isolated and culturally diverse, Tyrolian sample of 131 suicides matched with 131 living psychiatric controls (Tables 1 and 2). Suicide clearly has major socio-cultural influences as highlighted by very different suicide rates among northern versus southern and western versus eastern European countries [34,35]. Such variance in suicide rates appeared in differences found in this study among subcultures within South Tyrol, such that subjects of the predominant Italian culture were less prevalent among suicides than controls, compared with members of Austro-German, Ladin, or other minority cultures, despite differences in overall prevalence of these cultural subgroups (ranking Italian $>$ Austrian $>$ Ladin $>$ others by population, but Ladin $>$ Austro-German $>$ Italian by suicide rate, indicating that suicide risk was not an artifact of population size). Another important finding was that suicide cases were much less likely to have had recent contact with a clinician within the preceding month. Low rates of recent help-seeking among suicides has been noted previously $[3,36]$. The observed excess of previous suicidal behavior among suicide cases was an expected outcome [23], as was a greater prevalence of family history of suicidal behavior $[22,37,38]$.

We found no significant differences between suicides and nonsuicidal psychiatric controls in the distribution of DSM-IV-TR psychiatric diagnoses, nor was diagnosis an a 
priori outcome measure as all subjects were recently hospitalized psychiatric patients. Nevertheless, suicides tended more often to be diagnosed with a bipolar disorder and less with a nonaffective psychotic disorder (Table 1). This tendency accords with previous studies indicating especially high rates of suicides among persons with mood disorders $[7,39]$. Unexpectedly, however, lifetime substance abuse was less prevalent among suicide cases than in the nonsuicidal psychiatric controls, whereas it has usually been found to be a risk factor for suicide and overdosing is a common method of suicide [14,39].

Nevertheless, substance abuse may exert complex effects, including possible decreases in anxiety, depression and variable effects on impulsive behaviors [40].

We found evidence of childhood trauma in nearly $60 \%$ of the suicide cases compared to $40 \%$ of the controls, in accord with previous associations of physical or sexual abuse with suicide [24,41-44]. In addition, suicides had experienced recent stressful life-events, such as loss or separation, more often than controls (conditional OR=2.44). However, employment-related or financial distress was not found more often among suicide cases, contrary to expectation from some previous research $[45,46]$, which includes evidence that such stressors interact with predisposing vulnerabilities to suicide $[25,47]$.

\section{Limitations}

This pilot study is relatively small and limited by the largely retrospective and clinical nature of the information analyzed, by possible differences in the quality of information obtained for cases and controls, incomplete reporting of some of the factors considered, and by the unusual regional and cultural setting that may limit generalizations. Lack of precise matching of cases and controls for psychiatric diagnoses may have influenced the observed outcomes. Nevertheless, the observed association of several expected risk factors with suicide supports the adequacy of the methods employed. It may be that use of hospitalized controls biases toward more recent clinical contact than among suicides.

\section{Conclusions}

Both temporally proximal and distal risk factors were found to be associated with suicide in South Tyrol, including family history of suicidal behavior, early stressful experiences, 
ACCEPTED M/ANUSCRIPT

recent inter-personal adversities, and membership in nondominant cultural subgroups, as well as previous suicidal behavior and lack of recent clinical contact-most of which were expected from previous research. Unexpected findings included apparent lack of association of financial or occupation-related problems or of substance abuse among the suicides.

\section{References}

[1] Nock MK, Borges G, Bromet EJ, Cha CB, Kessler RC, Lee S. Suicide and suicidal behavior. Epidemiol Rev 2008;30:133-54. doi:10.1093/epirev/mxn002.

[2] WHO (World Health Organization). Preventing Suicide: A Global Imperative. Geneva: WHO, 2014.

[3] Simon RI, Hales RE. Textbook of suicide assessment and management (2nd ed.). Washington, DC: American Psychiatric Press; 2012.

[4] Franklin JC, Ribeiro JD, Fox KR, Bentley KH, Kleiman EM, Huang X, et al. Risk factors for suicidal thoughts and behaviors: A meta-analysis of 50 years of research. Psychol Bull 2017;143:187-232. doi:10.1037/bul0000084.

[5] Isometsä ET. Psychological autopsy studies-a review. Eur Psychiatry 2001;16:379-85.

[6] Cavanagh JTO, Carson AJ, Sharpe M, Lawrie SM. Psychological autopsy studies of suicide: a systematic review. Psychol Med 2003;33:395-405.

[7] Harris EC, Barraclough B. Excess mortality of mental disorder. Br J Psychiatry 1998;173:11-53. doi:10.1192/bjp.173.1.11.

[8] Hawton K, Casañas I Comabella C, Haw C, Saunders K. Risk factors for suicide in individuals with depression: a systematic review. J Affect Disord 2013;147:17-28. doi:10.1016/j.jad.2013.01.004.

[9] Tondo L, Pompili M, Forte A, Baldessarini RJ. Suicide attempts in bipolar disorders: Comprehensive review of 101 reports. Acta Psychiatr Scand 2016;133:174-86. doi:10.1111/acps.12517.

[10] Hendin H. Suicide: a review of new directions in research. Hosp Community Psychiatry 1986;37:148-54.

[11] Giupponi G, Bizzarri J, Pycha R, Innamorati M, Lester D, Conca A, et al. Socioeconomic risk factors and depressive symptoms in alcohol use disorders among male suicides in South Tirol, Italy. J Addict Dis 2010;29:466-74. doi:10.1080/10550887.2010.509272.

[12] Waern M. Alcohol dependence and misuse in elderly suicides. Alcohol 2003;38:249-54.

[13] Carrà G, Bartoli F, Crocamo C, Brady KT, Clerici M. Attempted suicide in people with cooccurring bipolar and substance use disorders: systematic review and meta-analysis. J Affect Disord 2014;167:125-35. doi:10.1016/j.jad.2014.05.066.

[14] Yuodelis-Flores C, Ries RK. Addiction and suicide: A review. Am J Addict 2015;24:98-104. 
doi:10.1111/ajad.12185.

[15] Ljung T, Chen Q, Lichtenstein P, Larsson H. Common etiological factors of attentiondeficit/hyperactivity disorder and suicidal behavior: a population-based study in Sweden. JAMA Psychiatry 2014;71:958-64. doi:10.1001/jamapsychiatry.2014.363.

[16] Pompili M, Innamorati M, Milelli M, Battuello M, Erbuto D, Lester D, et al. Temperaments in completed suicides: Are they different from those in suicide attempters and controls? Compr Psychiatry 2016;65:98-102. doi:10.1016/j.comppsych.2015.11.003.

[17] Vázquez GH, Gonda X, Lolich M, Tondo L, Baldessarini R. Suicidal risk and affective temperaments evaluated with TEMPS-A. Harv Rev Psychiatry 2017.

[18] Linehan M, Rizvi S, Welch S, Page B. Psychiatric disorder of suicidal behavior: personality disorders. In: Hawton K, van Heeringen C, editors. Int. Handb. Suicide Attempted Suicide, New York, NY: Wiley Press; 2000, p. 147-179.

[19] Pompili M. [Suicide Prevention (Italian)]. Bologna: Il Mulino Press; 2013.

[20] Baytunca MB, Ata E, Ozbaran B, Kaya A, Kose S, Aktas EO, et al. Childhood sexual abuse and supportive factors. Pediatr Int 2017;59:10-5. doi:10.1111/ped.13065.

[21] Martin MS, Dykxhoorn J, Afifi TO, Colman I. Child abuse and the prevalence of suicide attempts among those reporting suicide ideation. Soc Psychiatry Psychiatr Epidemiol 2016;51:1477-84. doi:10.1007/s00127-016-1250-3.

[22] Baldessarini RJ, Hennen J. Genetics of suicide: an overview. Harv Rev Psychiatry 2004;12:1-13.

[23] Rudd MD. Suicidality in clinical practice: anxieties and answers. J Clin Psychol 2006;62:157-9. doi:10.1002/jclp.20219.

[24] Hawton K, van Heeringen K. Suicide. Lancet 2009;373:1372-81. doi:10.1016/S01406736(09)60372-X.

[25] Mann JJ, Waternaux C, Haas GL, Malone KM. Toward a clinical model of suicidal behavior in psychiatric patients. Am J Psychiatry 1999;156:181-9. doi:10.1176/ajp.156.2.181.

[26] Simon RI, Hales RE, American Psychiatric Publishing. The American Psychiatric Publishing textbook of suicide assessment and management. American Psychiatric Pub; 2012.

[27] Giupponi G, Conca A, Innamorati M, Forte A, Lester D, Erbuto D, et al. Differences among South Tyrolean suicides: a psychological autopsy study. Wien Klin Wochenschr 2016;128:125-30. doi:10.1007/s00508-015-0877-5.

[28] McGirr A, Séguin M, Renaud J, Benkelfat C, Alda M, Turecki G. Gender and risk factors for suicide: evidence for heterogeneity in predisposing mechanisms in a psychological autopsy study. $\mathrm{J}$ Clin Psychiatry 2006;67:1612-7.

[29] Innamorati M, Pompili M, Masotti V, Personé F, Lester D, Tatarelli R, et al. Completed versus attempted suicide in psychiatric patients: a psychological autopsy study. J Psychiatr Pract 2008;14:216-24. doi:10.1097/01.pra.0000327311.04153.01.

[30] Pycha R, Pompili M, Innamorati M, Schwitzer J, Lester D, Sani G, et al. Sex and ethnic differences 
among South Tirolean suicides: A psychological autopsy study. Eur Psychiatry 2009;24:47-56.

[31] Giupponi G, Bizzarri J, Pycha R, Innamorati M, Lester D, Conca A, et al. Socioeconomic risk factors and depressive symptoms in alcohol use disorders among male suicides in South Tirol, Italy. J Addict Dis 2010;29:466-74. doi:10.1080/10550887.2010.509272.

[32] Spitzer RL, Williams JB, Gibbon M, First MB. The Structured Clinical Interview for DSM-III-R (SCID). I: History, rationale, and description. Arch Gen Psychiatry 1992;49:624-9. doi:10.1001/archpsyc.1992.01820080032005.

[33] Schafer JL. Multiple imputation: a primer. Stat Methods Med Res 1999;8:3-15. doi:10.1177/096228029900800102.

[34] Baldessarini RJ, Tondo L, Strombom IM, Dominguez S, Fawcett J, Licinio J, et al. Ecological studies of antidepressant treatment and suicidal risks. Harv Rev Psychiatry 2007;15:133-45. doi:10.1080/10673220701551102.

[35] World Health Organization (WHO). Preventing Suicide: a Global Imperative. Geneva: WHO, 2014.

[36] Sveticic J, Milner A, De Leo D. Contacts with mental health services before suicide: a comparison of Indigenous with non-Indigenous Australians. Gen Hosp Psychiatry 2012;34:185-91. doi:10.1016/j.genhosppsych.2011.10.009.

[37] Barraclough B, Bunch J, Nelson B, Sainsbury P. A hundred cases of suicide: clinical aspects. Br J Psychiatry 1974;125:355-73.

[38] Hawton K, van Heeringen K. Suicide. Lancet 2009;373:1372-81. doi:10.1016/S01406736(09)60372-X.

[39] Arsenault-Lapierre G, Kim C, Turecki G. Psychiatric diagnoses in 3275 suicides: a meta-analysis. BMC Psychiatry 2004;4:37. doi:10.1186/1471-244X-4-37.

[40] Bolton JM, Robinson J, Sareen J. Self-medication of mood disorders with alcohol and drugs in the National Epidemiologic Survey on Alcohol and Related Conditions. J Affect Disord 2009;115:36775. doi:10.1016/j.jad.2008.10.003.

[41] Farber EW, Herbert SE, Reviere SL. Childhood abuse and suicidality in obstetrics patients in a hospital-based urban prenatal clinic. Gen Hosp Psychiatry 1996;18:56-60.

[42] Szyf M. Early life, the epigenome and human health. Acta Paediatr 2009;98:1082-4. doi:10.1111/j.1651-2227.2009.01382.x.

[43] Szyf M. The early life environment and the epigenome. Biochim Biophys Acta 2009;1790:878-85. doi:10.1016/j.bbagen.2009.01.009.

[44] McGowan PO, Sasaki A, D’Alessio AC, Dymov S, Labonté B, Szyf M, et al. Epigenetic regulation of the glucocorticoid receptor in human brain associates with childhood abuse. Nat Neurosci 2009;12:342-8. doi:10.1038/nn.2270.

[45] Turecki G, Ernst C, Jollant F, Labont B, Mechawar N. The neurodevelopmental origins of suicidal behavior. Trends Neurosci 2012;35:14-23. doi:10.1016/j.tins.2011.11.008.

[46] Kerr WC, Kaplan MS, Huguet N, Caetano R, Giesbrecht N, McFarland BH. Economic Recession, 


\section{ACCEPTED MANUSCRIPT}

Alcohol, and Suicide Rates: Comparative Effects of Poverty, Foreclosure, and Job Loss. Am J Prev Med 2017;52:469-75. doi:10.1016/j.amepre.2016.09.021.

[47] Andrews JA, Lewinsohn PM. Suicidal attempts among older adolescents: prevalence and cooccurrence with psychiatric disorders. J Am Acad Child Adolesc Psychiatry 1992;31:655-62. doi:10.1097/00004583-199207000-00012. 
Table 1. Factors associated with suicide cases versus psychiatric controls

\begin{tabular}{|c|c|c|c|c|c|}
\hline Factors & Suicides & Nonsuicides & $\begin{array}{l}\text { Conditional } \\
\text { Odds Ratio }\end{array}$ & $\chi^{2}$ & $p$-value \\
\hline Subjects (n) & 131 & 131 & 1.00 & matched & - \\
\hline $\operatorname{Men}(\%)$ & 61.8 & 61.8 & 1.00 & matched & - \\
\hline Age \pm SD (years) & $48.2 \pm 15.5$ & $48.3 \pm 15.4$ & 1.00 & matched & - \\
\hline Family suicidal risk (\%) & 27.4 & 8.3 & 2.86 & 5.33 & 0.02 \\
\hline Interpersonal stressors (\%) ${ }^{\mathbf{a}}$ & 48.9 & 33.6 & 2.44 & 8.80 & 0.003 \\
\hline Childhood trauma (\%) & 34.4 & 22.1 & 2.40 & 7.84 & 0.005 \\
\hline No clinician $\leq 4$ weeks $(\%)$ & 48.1 & 26.0 & 2.33 & 5.63 & 0.02 \\
\hline Suicide attempts $(\%)$ & 25.2 & 15.3 & 2.25 & 5.03 & 0.03 \\
\hline Italian ethnicity $(\%)^{\mathbf{b}}$ & 11.5 & 21.4 & 0.46 & 4.11 & 0.04 \\
\hline Educated $\leq 8$ years $(\%)$ & 63.4 & 58.0 & 1.41 & 0.88 & 0.35 \\
\hline Living alone (\%) & 23.7 & 29.0 & 0.90 & 0.03 & 0.87 \\
\hline Unemployed (\%) & 44.3 & 48.9 & 0.83 & 0.30 & 0.58 \\
\hline Unmarried (\%) & 59.5 & 70.2 & 0.72 & 0.98 & 0.32 \\
\hline $\begin{array}{l}\text { Diagnosis }(\%) \\
\text { Bipolar disorder } \\
\text { Substance abuse } \\
\text { Major depression } \\
\text { Other } \\
\text { Psychosis }\end{array}$ & $\begin{array}{l}20.3 \\
31.1 \\
20.3 \\
17.6 \\
10.8\end{array}$ & $\begin{array}{l}13.0 \\
29.8 \\
19.1 \\
21.4 \\
16.8\end{array}$ & $\begin{array}{l}1.50 \\
1.08 \\
1.00 \\
0.91 \\
0.64\end{array}$ & $0.54^{\mathrm{c}}$ & 0.59 \\
\hline
\end{tabular}

a. Recent ( $\leq 6$ months) romantic failure or family distress or discord.

b. Ethnic suicide risks: Ladin $66.7 \%>$ Austro-German $51.0 \%>$ Italian $34.9 \%$.

c. Marginal homogeneity test. 
Table 2. Multivariate conditional logistic regression modeling for factors associated with suicides versus psychiatric controls

\begin{tabular}{|l|c|c|c|}
\hline Factor & $\begin{array}{c}\text { Slope } \\
(\beta)\end{array}$ & $\begin{array}{c}\text { Odds Ratio } \\
{[\mathbf{9 5 \%} \text { CI] }}\end{array}$ & $\boldsymbol{p}$-value \\
\hline Non-Italian ethnicity & 1.21 & $3.36[1.35-8.37]$ & 0.01 \\
\hline Interpersonal distress & 1.00 & $2.70[1.35-5.40]$ & 0.01 \\
\hline Family suicidal history & 0.98 & $2.67[1.10-6.50]$ & 0.03 \\
\hline No recent clinical contact & 0.95 & $2.57[1.22-5.44]$ & 0.02 \\
\hline Childhood trauma & 0.93 & $2.54[1.22-5.23]$ & 0.01 \\
\hline
\end{tabular}

Fit statistics: $-2 \log$ Likelihood $(-2 L L)=76.31 ; \chi^{2}[\mathrm{df}=4]=17.4 ; p=0.008$ 